\title{
Generation, Visualization, and Editing of 3D Video
}

\author{
Takashi Matsuyama and Takeshi Takai \\ Graduate School of Informatics, Kyoto University \\ Yoshidahonmachi, Sakyo-ku, Kyoto, 606-8501, Japan \\ tm@i.kyoto-u.ac.jp, takesi-t@vision.kuee.kyoto-u.ac.jp
}

\begin{abstract}
$3 D$ video is the ultimate image medium recording dynamic visual events in the real world as is. Recorded object behaviors can be observed from any viewpoint, because 3D video records the object's full 3D shape, motion, and precise surface properties (i.e. color and texture). In our last paper[1], we presented a method of reconstructing dynamic $3 D$ object shape from multi-view video images, by which a temporal series of $3 D$ voxel representations of the object behavior can be obtained in real-time. In this paper, following an overview of the real-time $3 D$ shape reconstruction method, we present 1) an algorithm of generating texture on the 3D object surface from the multi-view video images, and 2) an editing system for visualizing $3 D$ video with an omnidirectional background image using versatile $3 D$ camera works. This paper mainly discusses how we can generate high fidelity object images from arbitrary viewpoints based on the $3 D$ object shape of limited accuracy. We propose a novel texture mapping algorithm which maps textures onto the $3 D$ object surface depending on a viewpoint. Experimental results demonstrate its effectiveness in generating high fidelity object images from arbitrary viewpoints.
\end{abstract}

\section{Introduction}

$3 \mathrm{D}$ video is the ultimate image medium recording dynamic visual events in the real world as is: time varying $3 \mathrm{D}$ object shape with high fidelity surface properties (i.e. color and texture). Its applications cover wide varieties of personal and social human activities: entertainment (e.g. 3D game and 3D
TV), education (e.g. 3D animal picture books), sports (e.g. sport performance analysis), medicine (e.g. 3D surgery monitoring), culture (e.g. 3D archive of traditional dance) and so on.

In recent years, several research groups developed real-time 3D shape reconstruction systems for 3D video and have opened up the new world of image media [1] [2] [3] [4] [5]. All these systems focus on capturing human body actions and share a group of distributed video cameras for real-time synchronized multi-viewpoint action observation. While the realtimeness of the earlier systems[4] [5] was confined to the synchronized multi-viewpoint video observation, parallel volume intersection on a PC cluster has enabled the real-time 3D shape reconstruction [1] [2] [3]. Our PC cluster system[1], for example, can reconstruct dynamic human 3D shape at about 10 frame per second in $2 \mathrm{~cm} \times 2 \mathrm{~cm} \times 2 \mathrm{~cm}$ voxel resolution without using any special hardwares or MMX instructions.

To cultivate the $3 \mathrm{D}$ video world and make it usable in everyday life, we have to solve the following technical problems:

Computation Speed : We have to develop both faster machines and algorithms, because near framerate $3 \mathrm{D}$ shape reconstruction has been attained only in coarse resolution and moreover texture mapping onto the reconstructed 3D shape is still done off-line.

High Fidelity : To obtain realistic 3D video in the same quality as ordinary video images, we have to develop high fidelity texture mapping methods as well as increase the resolution.

Wide Area Observation : 3D areas observable by the systems developed so far are confined to about 
$2 m \times 2 m \times 2 m$, which should be extended considerably to capture human actions like sports playing.

Data Compression : Since naive representation of $3 \mathrm{D}$ video results in huge data, effective compression methods are required to store and transmit $3 \mathrm{D}$ video data.

Editing and Visualization : Since editing and visualization of 3D video are conducted in the 4D space (3D geometric $+1 \mathrm{D}$ temporal), we have to develop human-friendly 3D video editors and visualizers that help a user to understand dynamic events in the $4 \mathrm{D}$ space.

In this paper, following an overview of the realtime 3D shape reconstruction method proposed in our last paper[1], we present 1) an algorithm of generating texture on the 3D object surface from the multi-view video images, and 2) an editing system for visualizing 3D video with an omnidirectional background image using versatile 3D camera works. This paper mainly discusses how we can generate high fidelity object images from arbitrary viewpoints based on the 3D object shape of limited accuracy. We propose a novel texture mapping algorithm which maps textures onto the $3 \mathrm{D}$ object surface depending on a viewpoint. Experimental results demonstrate its effectiveness in generating high fidelity object images from arbitrary viewpoints.

\section{Real-Time 3D Object Behavior Reconstruc- tion System}

\subsection{System Organization}

Figure 1 illustrates the hardware organization of our real-time active $3 \mathrm{D}$ object behavior reconstruction system. It consists of

- PC cluster: 16 node PCs (dual Pentium III $600 \mathrm{MHz}$ ) are connected through Myrinet, an ultra high speed network (full duplex 1.28Gbps). PM library for Myrinet PC clusters[6] allows very low latency and high speed data transfer, based on which we can implement efficient parallel processing on the PC cluster.

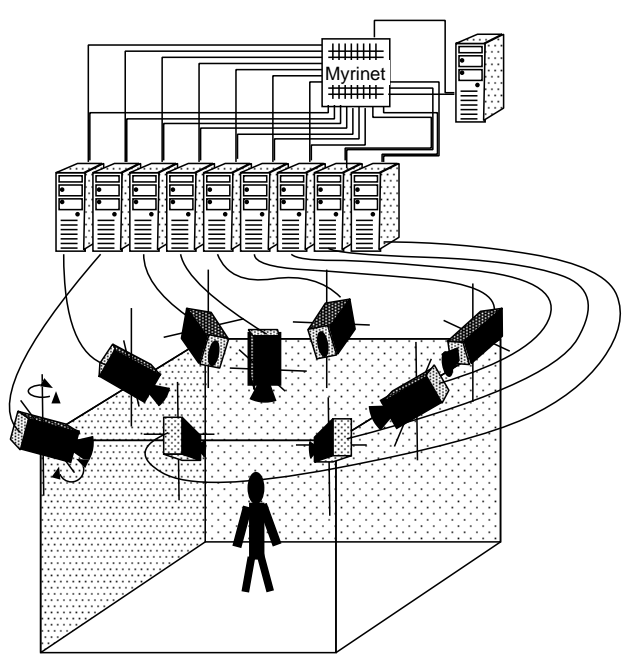

Figure 1. PC cluster for real-time active 3D object behavior reconstruction system.

- Distributed active video cameras: Among 16, 12 PCs have fixed-viewpoint pan-tilt-zoom (FVPTZ) cameras[7], respectively, for active object tracking and image capturing. In the FV-PTZ camera, the projection center stays fixed irrespectively of any camera rotations and zoomings, which greatly facilitates real-time active object tracking and 3D shape reconstruction.

\subsection{Basic Method of 3D Video Generation}

Figure 2 illustrates the basic process of generating a $3 \mathrm{D}$ video frame in our system:

1. Synchronized Multi-Angle Image Acquisition: A set of multi-viewpoint object images are taken simultaneously by a group of distributed video cameras (Figure 2 top row).

2. Silhouette Extraction: Background subtraction is applied to each captured image to generate a set of multi-viewpoint object silhouettes (Figure 2 second top row).

3. Silhouette Volume Intersection: Each silhouette is back-projected into the common 3D space to generate a visual cone encasing the 3D object. Then, such 3D cones are intersected with each other to generate the voxel representation of the object shape (Figure 2 middle). 

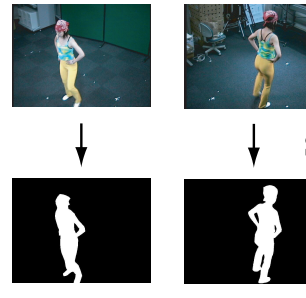

$\downarrow$ Silhouette Extraction
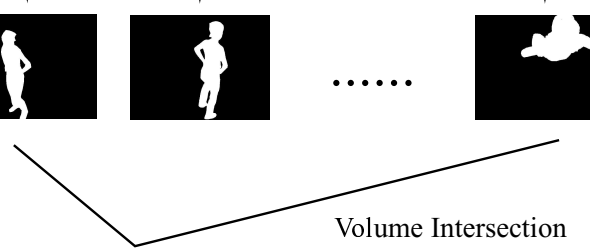

Volume Intersection

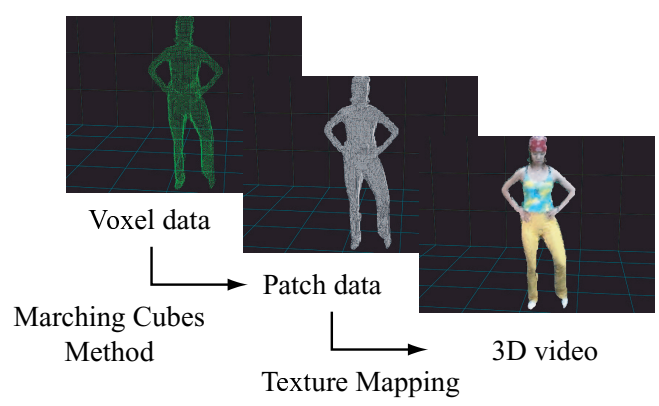

Figure 2. 3D video capturing process

4. Surface Shape Computation: The discrete marching cubes method[8] is applied to convert the voxel representation to the surface patch representation (Figure 2 second bottom).

5. Texture Mapping: Color and texture on each patch are computed from the observed images (Figure 2 bottom).

By repeating the above process for each video frame, we have a live 3D motion picture.

\subsection{Parallel Volume Intersection Algorithm Using Plane-to-Plane Perspective Projection}

The back-projection is the most expensive computation in the above volume intersection method, because it involves a considerable amount of arithmetic operations. To accelerate the computation, we first developed the plane-to-plane perspective projection (PPPP) algorithm, where the $3 \mathrm{D}$ voxel space is partitioned into a group of parallel planes and the cross-section of the 3D object volume on each plane is reconstructed.

Figure 3 shows the basic algorithm, where the backprojection process is divided into the following two stages:

1. First an object silhouette in the image plane is back-projected on the base plane in the 3D space.
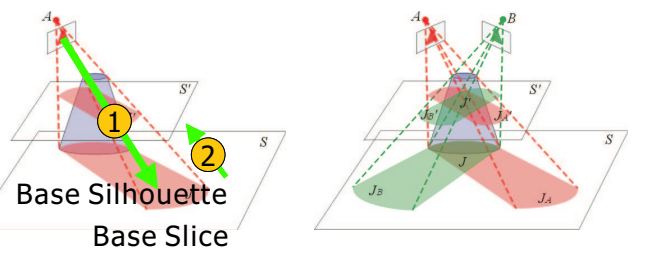

Figure 3. Plane-to-plane perspective projection algorithm

2. Then the back-projected base silhouette is projected onto each of the parallel planes.

Finally, back-projected silhouettes on each plane are intersected with each other to generate an object crosssection on that plane. By stacking up such crosssections, we have the voxel representation of the 3D object shape.

From a computational viewpoint, the plane-to-plane perspective projection is much less computationally expensive than general 3D perspective projection.

The next step to realize real-time 3D shape reconstruction is to introduce parallel processing by the PC cluster. Figure 4 illustrates the processing flow of the parallel pipelined PPPP algorithm:

1. Image Capture : Triggered by a capturing command, each PC with a camera captures a video frame, by which 12 synchronized multiviewpoint images are acquired (Figure 4 top row).

2. Silhouette Extraction : Each PC extracts an object silhouette from the video frame (Figure 4 second top row).

3. Projection to the Base-Plane Each PC projects the silhouette onto the common base-plane in the 3D space (Figure 4 third top row).

4. Base-Plane Silhouette Duplication : All baseplane silhouettes are duplicated across 16 PCs over the network so that each PC has the full set of 12 base-plane silhouettes (Figure 4 forth top row).

5. Object Cross Section Computation : Each of 16 PCs computes object cross sections on specified parallel planes in parallel (Figure 4 three bottom rows). 


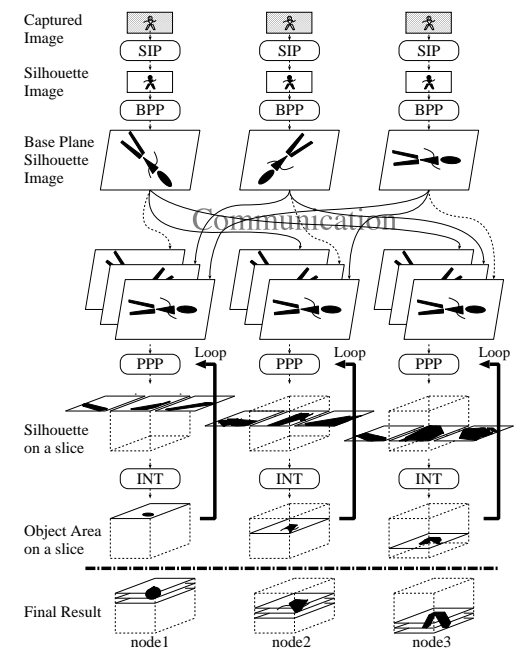

Figure 4. Processing flow of the parallel pipelined 3D shape reconstruction.

The above processing is implemented as the 5 stage pipeline process as well as stages $1,2,3$, and 5 are executed in parallel by the PCs. Note that although stage 4 involves communications among 16 PCs, its overhead time is limited because the transmitted data are just bit maps of small size representing the baseplane silhouettes and the speed of the network is very high.

\subsection{Performance Evaluation}

In the experiments of the 3D volume reconstruction, we used just 9 PCs with 9 active cameras placed at the ceiling (Figure 1). This is because the floor, which is regarded as the base-plane, can be commonly observed from all the cameras.

Table 1 shows the performance of the real-time 3D object behavior reconstruction. Since we employ pipeline processing, the throughput time is shorter than the total elapsed time. The pipeline effect is shown as "Pipeline Factor" and the average volume reconstruction rate as "Volume/sec.". While the pipeline factor can be regarded as constant, the volume reconstruction rate increases gradually as the voxel resolution decreases. Note that the volume reconstruction rate is saturating. This is because the image capture and silhouette extraction stages spend constant processing time irrespectively of the voxel resolution.

\begin{tabular}{|l|l|l|l|l|}
\hline $\begin{array}{l}\text { Voxel } \\
\text { Size }\end{array}$ & $\begin{array}{l}\text { Total } \\
\text { Elapsed } \\
\text { time }\end{array}$ & $\begin{array}{l}\text { Throughput } \\
\text { Time }\end{array}$ & $\begin{array}{l}\text { Volume } \\
/ \text { sec. }\end{array}$ & $\begin{array}{l}\text { Pipeline } \\
\text { Factor }\end{array}$ \\
\hline \hline $1 \mathrm{~cm}$ & $651.019 \mathrm{~ms}$ & $564.413 \mathrm{~ms}$ & 1.77 & 1.15 \\
\hline $2 \mathrm{~cm}$ & $145.127 \mathrm{~ms}$ & $114.065 \mathrm{~ms}$ & 8.77 & 1.27 \\
\hline $3 \mathrm{~cm}$ & $107.031 \mathrm{~ms}$ & $80.873 \mathrm{~ms}$ & 12.37 & 1.32 \\
\hline $5 \mathrm{~cm}$ & $80.484 \mathrm{~ms}$ & $64.013 \mathrm{~ms}$ & 15.62 & 1.25 \\
\hline
\end{tabular}

Table 1. Processing speed. (Total Elapsed Time: sum of the elapsed times at all stages, Throughput Time: time interval between the outputs, Volume/sec.: number of volumes reconstructed per second, Pipeline Factor=(Total Elapsed Time)/(Throughput Time).)
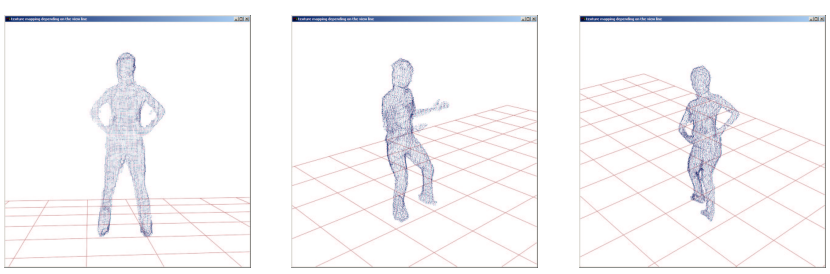

Figure 5. Voxel representations of a 3D object behavior

\section{High Fidelity Texture Mapping Algorithm}

Figure 5 illustrates a partial sequence of 3D voxel data of a dancing person at the resolution of $1 \mathrm{~cm} \times$ $1 \mathrm{~cm} \times 1 \mathrm{~cm}$. Then we apply to each voxel data the discrete marching cubes method[8] to convert the 3D object shape into the triangular patch representation. And finally we map textures extracted from the observed multi-viewpoint video onto the patches to generate a frame of 3D video. These processes are applied as a post-process after generating the $3 \mathrm{D}$ voxel data.

In this section, we propose a novel texture mapping algorithm to generate high fidelity 3D video. The problem we are going to solve here is how we can generate high fidelity object images from arbitrary viewpoints based on the 3D object shape of limited accuracy. That is, computed 3D voxel data are just approximation of real 3D object shape (i.e. all concavities cannot be reconstructed) and include considerable amount of noise. Moreover, outward normals of triangular patches computed by the discrete matching cubes method are roughly quantized at the resolution of 45 degree. 


\subsection{Naive Algorithm: Viewpoint Independent Patch-Based Method}

We first implemented a naive texture mapping algorithm, which selects the most "appropriate" camera for each patch and then maps onto the patch the texture extracted from the image observed by the selected camera. Since this texture mapping is conducted independently of the viewer's viewpoint of 3D video, we call it as the Viewpoint Independent Patch-Based Method (VIPBM in short).

Algorithm (Figure 6)

1. For each patch $p_{i}$, do the following processing.

2. Compute the locally averaged normal vector $V_{l m n}$ using normals of $p_{i}$ and its neighboring patches.

3. For each camera $c_{j}$, compute viewline vector $V_{c_{j}}$ directing toward the centroid of $p_{i}$.

4. Select such camera $c^{*}$ that the angle between $V_{l m n}$ and $V_{c_{j}}$ becomes maximum.

5. Extract the texture of $p_{i}$ from the image captured by camera $c^{*}$.

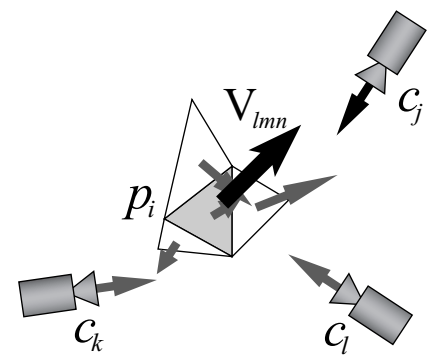

Figure 6. Viewpoint independent patch-based method

This method generates fully textured 3D object shape, which can be viewed from arbitrary viewpoints with ordinary 3D graphic display systems. Moreover, its data size is very compact compared with that of the original multi-viewpoint video data.

From the viewpoint of fidelity, however, the displayed image quality is not satisfiable;

1. Due to the rough quantization of patch normals, the best camera $c^{*}$ for a patch varies from patch to patch even if they are neighboring. Thus, textures on neighboring patches are often extracted from those images captured by different cameras (i.e. viewpoints), which introduces jitters in displayed images.

2. Since the texture mapping is conducted patch by patch and their normals are not accurate, textures of neighboring patches may not be smoothly connected. This introduces jitters at patch boundaries in displayed images.

To overcome these quality problems, we developed a viewpoint dependent vertex-based texture mapping algorithm. In this algorithm, the color (i.e. RGB values) of each vertex of patches is computed taking into account of the viewpoint of a viewer and then the texture of each patch is generated by interpolating color values of its three vertices. In what follows, we first define words and symbols to describe the algorithm and then present the computation process, followed by experimental results.

\subsection{Viewpoint Dependent Vertex-Based Texture Mapping Algorithm}

\section{(1) Definitions}

First of all, we define words and symbols as follows (Figure 7), where bold face symbols denote 3D position/directive vectors:

- a group of cameras: $C=\left\{c_{1}, c_{2}, \ldots, c_{n}\right\}$

- a viewpoint for visualization: eye

- a set of surface patches: $P=\left\{p_{1}, p_{2}, \ldots, p_{m}\right\}$

- outward normal vector of patch $p_{i}: \boldsymbol{n}_{p_{i}}$

- a viewing direction from eye toward the centroid of $p_{i}: \boldsymbol{v}_{\text {eye } \rightarrow p_{i}}$

- a viewing direction from $c_{j}$ toward the centroid of $p_{i}: \boldsymbol{v}_{c_{j} \rightarrow p_{i}}$

- vertices of $p_{i}: \mathbf{v}_{p_{i}}^{k} \quad(k=1,2,3)$

- vertex visible from $c_{j}$ (defined later): $\mathbf{v}_{p_{i}, c_{j}}^{k}$

- RGB values of $\mathbf{v}_{p_{i}, c_{j}}^{k}$ (defined later): $I\left(\mathbf{v}_{p_{i}, c_{j}}^{k}\right)$ 


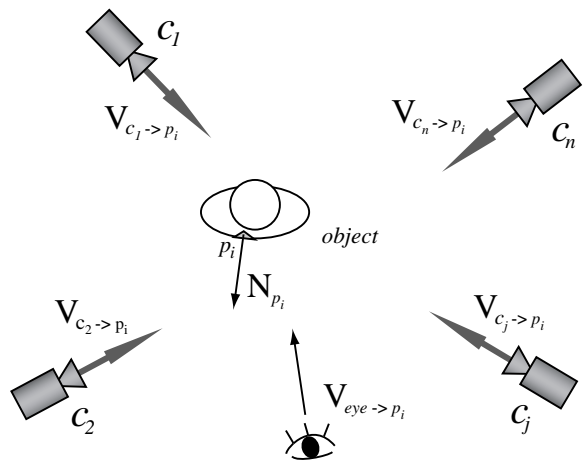

Figure 7. Viewpoint and camera position

- a depth buffer of $c_{j}: \mathbf{B}_{c_{j}}$

Geometrically this buffer is the same as the image plane of camera $c_{j}$. Each pixel of $\mathbf{B}_{c_{j}}$ records such patch ID that is nearest from $c_{j}$ as well as the distance to that patch from $c_{j}$ (Figure 8). When a vertex of a patch is mapped onto a pixel, its vertex ID is also recorded in that pixel.

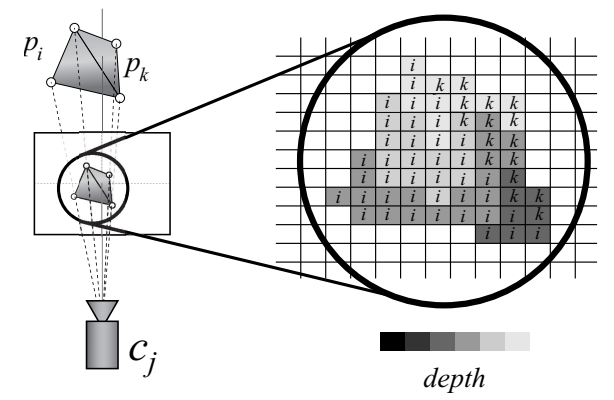

Figure 8. Depth buffer

(2) Visible Vertex from Camera $c_{j}$

The vertex visible from $c_{j} \mathbf{v}_{p_{i}, c_{j}}^{k}$ is defined as follows.

1. The face of patch $p_{i}$ can be observed from camera $c_{j}$, if the following condition is satisfied.

$$
\boldsymbol{n}_{p_{i}} \cdot \boldsymbol{v}_{c_{j} \rightarrow p_{i}}<0
$$

2. $\mathbf{v}_{p_{i}}^{k}$ is not occluded by any other patches.

Then, we can determine $\mathbf{v}_{p_{i}, c_{j}}^{k}$ by the following process:

1. First, project all the patches that satisfy equation (1) onto the depth buffer $B_{c_{j}}$.

2. Then, check the visibility of each vertex using the buffer. Figure 9 illustrates possible spatial configurations between a pair of patches: all the vertices in type (1) and (2) are visible, while in type (5) three vertices of the occluded patch are not visible. In type (3) and (4), only some vertices are visible.

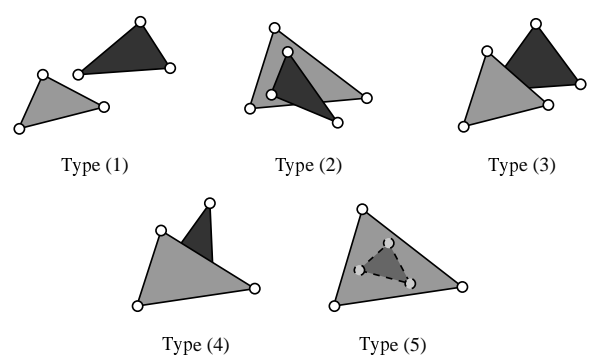

Figure 9. Relations between patches

RGB values $I\left(\mathbf{v}_{p_{i}, c_{j}}^{k}\right)$ of the visible vertex $\boldsymbol{v}_{p_{i}, c_{j}}^{k}$ are computed by

$$
I\left(\mathbf{v}_{p_{i}, c_{j}}^{k}\right)=I_{c_{j}}\left(\hat{\mathbf{v}}_{p_{i}, c_{j}}\right),
$$

where $I_{c_{j}}(\mathbf{v})$ shows RGB values of pixel $\mathbf{v}$ on the image captured by camera $c_{j}$, and $\hat{\mathbf{v}}_{p_{i}, c_{j}}^{k}$ denotes the pixel position onto which the vertex $\mathbf{v}_{p_{i}, c_{j}}^{k}$ is mapped by the imaging process of camera $c_{j}$.

\section{(3) Algorithm}

1. Compute RGB values of all vertices visible from each camera in $C=\left\{c_{1}, c_{2}, \ldots, c_{n}\right\}$.

2. Specify the viewpoint eye.

3. For each surface patch $p_{i} \in P$, do 4 to 9 .

4. If $\boldsymbol{v}_{\text {eye } \rightarrow p_{i}} \cdot \boldsymbol{n}_{p_{i}}<0$, then do 5 to 9 .

5. Compute weight $w_{c_{j}}=\left(\boldsymbol{v}_{c_{j} \rightarrow p_{i}} \cdot \boldsymbol{v}_{\text {eye } \rightarrow p_{i}}\right)^{m}$, where $m$ is a weighting factor to be specified a priori.

6. For each vertex $\mathbf{v}_{p_{i}}^{k}(k=1,2,3)$ of patch $p_{i}$, do 7 to 8 .

7. Compute the normalized weight for $\mathbf{v}_{p_{i}}^{k}$ by

$$
\bar{w}_{c_{j}}^{k}=\frac{w_{c_{j}}^{k}}{\sum_{l} w_{c_{l}}^{k}} .
$$

Here, if $\mathbf{v}_{p_{i}}^{k}$ is visible from camera $c_{j}$, then $w_{c_{j}}^{k}=$ $w_{c_{j}}$, else $w_{c_{j}}^{k}=0$.

8. Compute the RGB values $I\left(\mathbf{v}_{p_{i}}^{k}\right)$ of $\mathbf{v}_{p_{i}}^{k}$ by

$$
I\left(\mathbf{v}_{p_{i}}^{k}\right)=\sum_{j=1}^{n} \bar{w}_{c_{j}}^{k} I\left(\mathbf{v}_{p_{i}, c_{j}}^{k}\right)
$$


9. Generate the texture of patch $p_{i}$ by linearly interpolating RGB values of its vertices. To be more precise, depending on the number of vertices with non-zero RGB values, the following processing is conducted:

- 3.

Generate RGB values at each point on the patch by linearly interpolating the RGB values of 3 vertices.

- 2 .

Compute mean values of the RGB values of the 2 vertices, which is regarded as those of the other vertex. Then apply the linear interpolation on the patch.

- 1 .

Paint the patch by the RGB values of the vertex.

- 0 .

Texture of the patch is not generated: painted by black for example.

By the above process, an image representing an arbitrary view (i.e from eye) of the 3D object is generated.

\subsection{Performance Evaluation}

To evaluate the performance of the proposed viewpoint dependent vertex-based method (VDVBM), we first compare it with the viewpoint independent patchbased method (VIPBM) qualitatively. Figures 10 and 11 show images generated by VIPBM and VDVBM, respectively, for the same 3D patch data. We can observe that VIPBM introduces many jitters in images, which are considerably reduced by VDVBM.
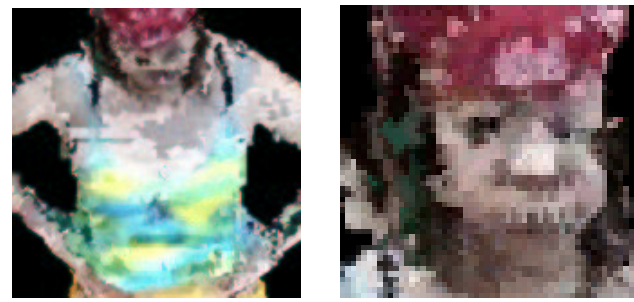

Figure 10. Images generated by the Viewpoint Independent Patch-Based Method

Then, we conducted quantitative performance evaluations. That is, we calculate RGB root-mean-square
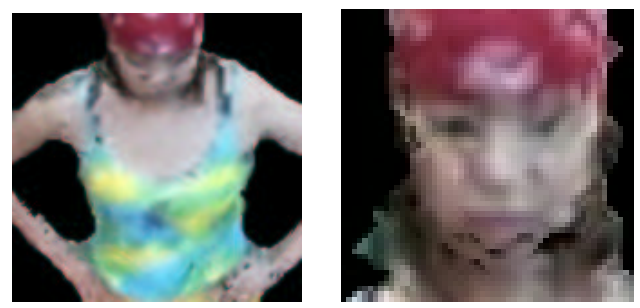

Figure 11. Images generated by the Viewpoint Dependent Vertex-Based Method

(rms) errors between a real image captured by camera $c_{j}$ and its corresponding images generated by VIPBM and VDVBM, respectively: in generating the images, the position and direction of camera $c_{j}$ are used as those of the viewpoint for the 3D video (i.e. eye in VDVBM). To evaluate the performance of VDVBM, we employed two methods: VDVBM-1 generates images including real images captured by camera $c_{j}$ itself, while VDVBM-2 excludes such real images captured by camera $c_{j}$. The experiments were conducted under the following settings:

- camera configuration: Figure 12

- image size: $640 \times 480$ [pixel] 24 bit RGB color

- viewpoint: camera 5

- weighting factor in VDVBM: $m=5$

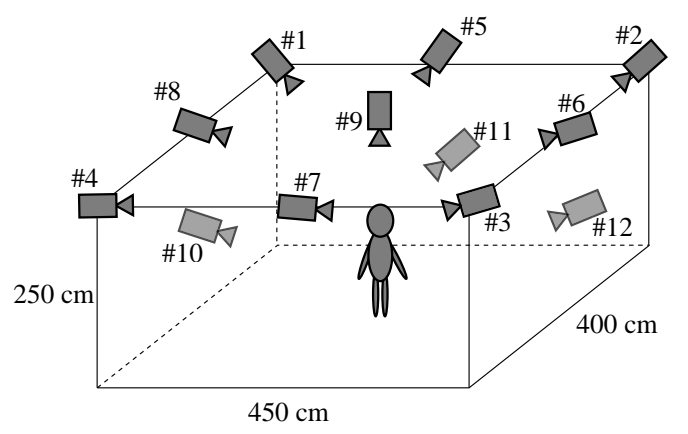

Figure 12. Camera Setting

Figure 13 illustrates the experimental results, where rms errors for frame 95 to 145 are computed. This figure proves that VDVBM performs better than VIPBM. The superiority of VDVBM and its high fidelity image generation capability can be easily observed in Figure 14 , where real and generated images for frame 110 and 120 are illustrated.

Finally, we tested how we can improve the performance of VDVBM by increasing the spatial resolution 


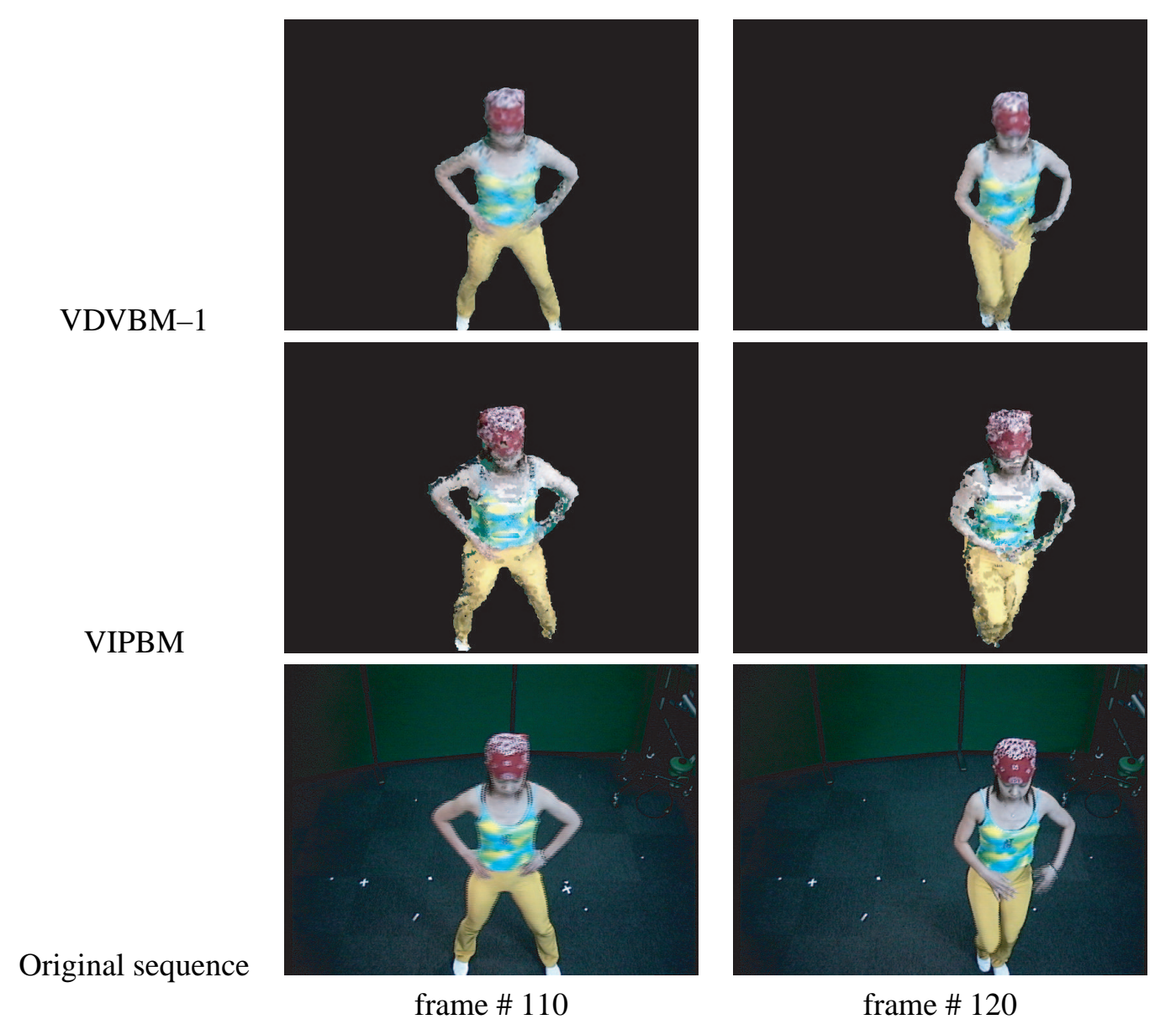

Figure 14. Sample images of generated 3D video

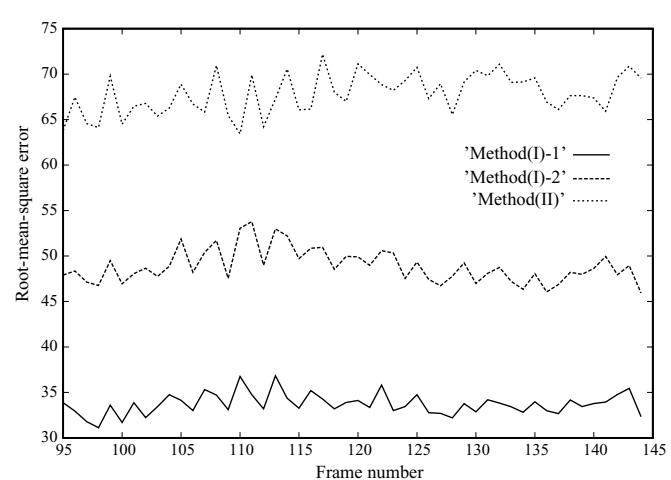

Figure 13. Root-mean-square error of RGB value (1)

of patch data. Figure 15 shows the method of subdividing a patch into three (S3) and six (S6) sub-patches to increase the spatial resolution.

Then, we examine the average side length of a patch on the image plane of each camera by projecting original and subdivided patches onto the image plane. Fig- ure 16 shows the mean side length in pixel on the image plane of each camera. Note that since camera 9 is located closer to the 3D object (see Figure 12), object images captured by it become larger than those by the other cameras, which caused bumps (i.e. larger side length in pixel) in the graphs in Figure 16.

We can observe that the spatial resolution of S6 is approximately the same as that of an observed image (i.e. 1 pixel). That is, S6 attains the finest resolution, which physically represents about $5 \mathrm{~mm}$ on the object surface. To put this in another way, we can increase the spatial resolution up to the six sub-division, which can improve the quality of images generated by VDVBM.

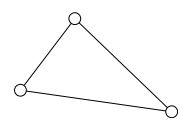

An original patch

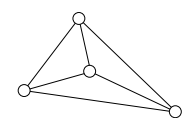

A patch subdivided into three (S3)

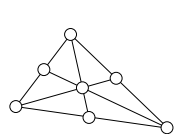

A patch subdivided into six (S6)

\section{Figure 15. Subdivision of a surface patch}




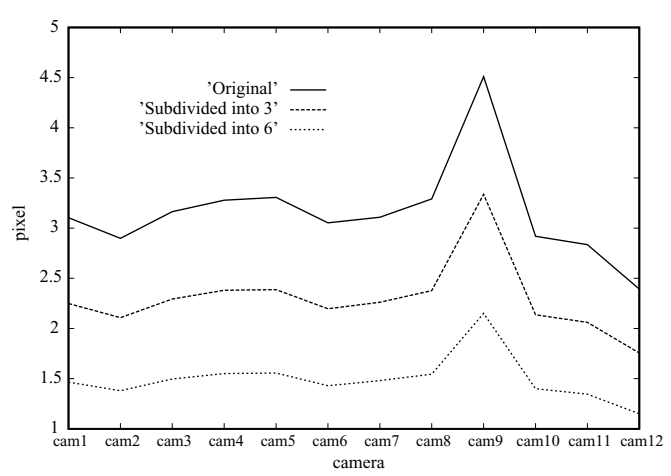

Figure 16. Mean side length in pixel on image planes of cameras

To quantitatively evaluate the quality archived by using subdivided patches, we calculated root-meansquare errors between real images and images generated by VDVBM-1 with original, S3, and S6, respectively. The root-mean-square errors are shown in Figure 17.

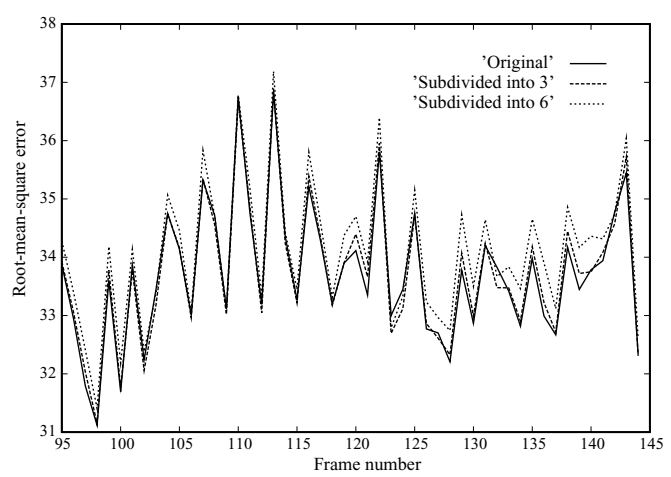

Figure 17. Root-mean-square errors of RGB value (2)

Figure 17 shows that subdividing patches does not numerically reduce the errors. The reasons of this observation can be considered as follows. We see that most of the errors arise around the contour of the object and edges of texture (e.g. an edge between skin and clothes, etc.) (Figure 18), and they are difficult to be reduced by subdividing patches because they come from motion blur or asynchronization, i.e. capturing the images is not perfectly synchronized. The errors are thus not reduced in total even if they may be in some parts. Fidelity of generated images using subdivided patches, however, is definitely improved (Figure 19). Accordingly, subdividing patches is effective from fidelity point of view.

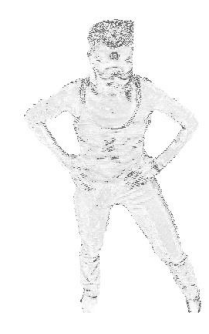

Figure 18. Subtraction between a real image and a generated image (frame \#106)

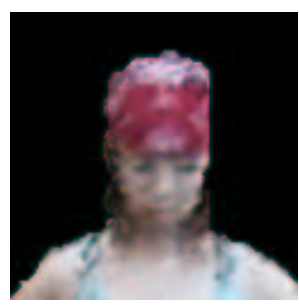

Original

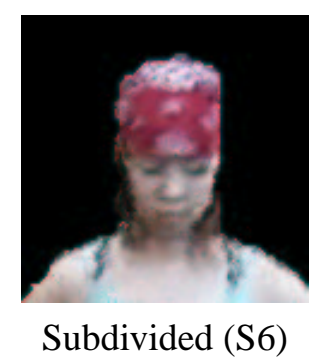

Figure 19. Example images visualized with original and subdivided patches (frame \#106)

Finally, we show examples generated by VDVBM1 with subdivided patches (S6) viewed from camera 5,11 , and an intermediate point between them (Figure 20 ). Figure 20 shows that the images generated by VDVBM look almost real even when they are viewed from the intermediate point of the cameras.

\section{Editing and Visualization System of 3D Video}

To visualize $3 \mathrm{D}$ video, we should introduce virtual cameras into the virtual scene where the reconstructed object is placed. A virtual background ${ }^{1}$ should be also introduced for discriminating between camera action and object motion.

In this situation, we have to specify many parameters to generate a camera-work that is utilized for visualization of 3D video:

- virtual camera parameters: viewpoint $x, y, z$; view direction $\psi, \phi$; angle of view $\theta$; focal length $f$, and so on.

\footnotetext{
${ }^{1}$ In our system, we use a dome-shaped omnidirectional background image as a virtual background. This image is generated by mosaicing multiple images taken by our FV-PTZ camera.
} 


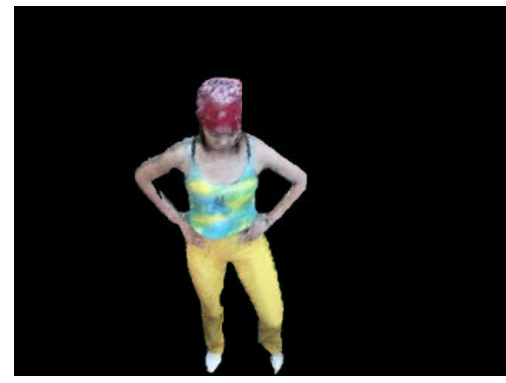

cam 5

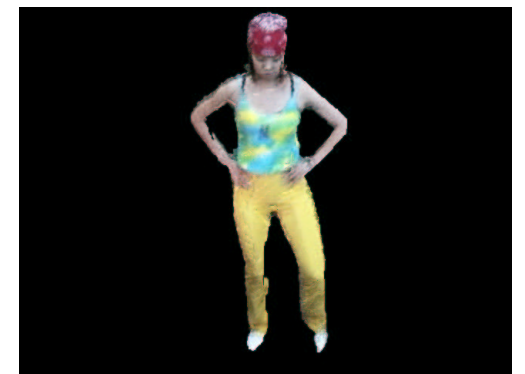

an intermediate image (cam 5 to cam 11)

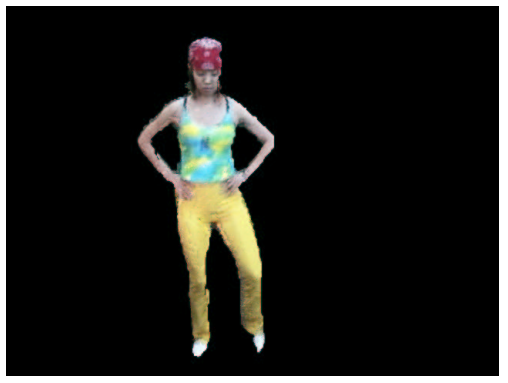

cam 11

Figure 20. Visualized 3D video with subdivided patches (frame\#103)

- object position, pose, and scale: $x_{o}, y_{o}, z_{o}$, $\psi_{o}, \phi_{o}, \rho_{o}, s_{o}$.

- background position, pose, and scale: $x_{b}, y_{b}, z_{b}$, $\psi_{b}, \phi_{b}, \rho_{b}, s_{b}$.

Furthermore, these parameters must be specified at each time, and they should be continuously changed for smooth visualization and natural camera-works.

To satisfy these requirements, we have devised two methods to generate camera-works as follows.

1. Key Frame Method: generating camera-works by temporal interpolation of parameters specified for arbitrary key frames.

\section{Automatic Camera-Work Generation} Method: generating camera-works by utilizing an object's parameters (e.g. height, direction).

These parameters are specified in the scene coordinate systems (Figure 21).

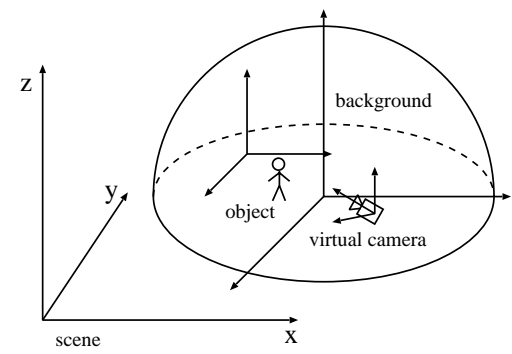

Figure 21. Virtual Scene Setup

\subsection{Key Frame Method (KFM)}

This method is often used in the ordinary computer graphics animation. Specifying the parameters (positions, rotations of a virtual camera, object, etc.) for arbitrary key frames allows us to temporally interpolate them to generate camera-works realizing such parameters (Figure 22).
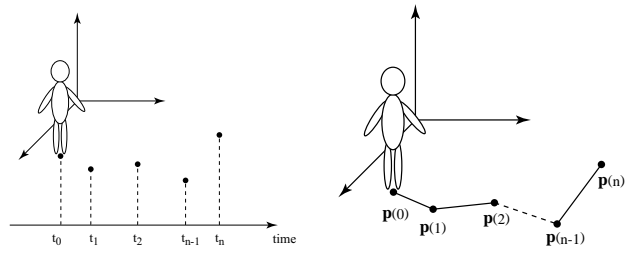

Figure 22. Temporal interpolation of parameters

\subsection{Automatic Camera-Work Generation Method (ACGM)}

To utilize KFM, a user has to consider framing of generated images while specifying parameters. For example, to generate a camera-work that keeps taking pictures of the object's front view, the user has to consider relations between the virtual camera and the object at each frame; this enforces a mount of effort upon the user.

To easily obtain camera-works such as described above, we propose a method for utilizing the object's parameters. The object generated by our volume intersection algorithm has the following parameters:

- position: the centroid of a group of voxels that form the object.

- height: length of the object along the $\mathrm{z}$ axis.

- direction: direction representing the front of the object. (This can be estimated from the voxel representation of the object. In this paper, we specify this beforehand.)

We remark that these parameters are defined under the assumption that the object is a standing human. Utilizing the object's parameters enables a user to obtain 
arbitrary camera-works. That is, the user has only to specify

1. framing of a picture: close up, middle shot, full shot, etc., and

\section{2. appearance of the object from the virtual cam-} era: front, back, etc.

Once the framing of a picture and the appearance of the object are specified, the parameters of the virtual camera are computed as follows:

- distance between the virtual camera and the object $d$ (Figure 23):

$$
d=\frac{r \cdot h}{2 \tan \frac{\theta}{2}},
$$

where $h$ is the height of the object. $\theta$ is an angle of view, which is arbitrarily specified. $r$ is the ratio of a size of an image to a size of the rendered object, and it is defined as follows:

- $r=1.0:$ full shot,

- $r=0.7$ : knee shot,

- $r=0.5$ : middle shot,

$-r=0.3:$ close shot, and

- $r=0.2$ : close up.

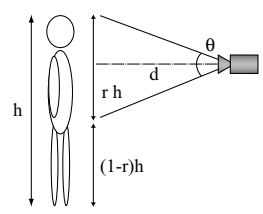

Figure 23. Relation between a virtual camera and an object (I)

- position of the virtual camera $\left(x_{c}, y_{c}, z_{c}\right)$ :

$$
\left\{\begin{array}{ll}
x_{c}=d \cos \phi \cos (\psi+\delta) & +x_{p} \\
y_{c}=d \cos \phi \sin (\psi+\delta) & +y_{p} \\
z_{c}=d \sin \phi & +z_{p}
\end{array},\right.
$$

where $d$ is given by Equation(5), and $\delta$ is an angle between the direction of the object and the $\mathrm{x}$ axis in the scene (Figure 24). $\phi$ and $\psi$ are arbitrarily specified (Figure 25). For example, to visualize the object's front view, $\psi$ and $\phi$ are set to be 0 , and an angle that a user desires, respectively. $\left(x_{p}, y_{p}, z_{p}\right)$ is, on the other hand, a gazing point of the virtual camera, where $x_{p}$ and $y_{p}$ are the same as the position of the object, and $z_{p}$ is given by

$$
z_{p}=h-\frac{h \cdot r}{2} \quad(0 \leq r \leq 1) .
$$

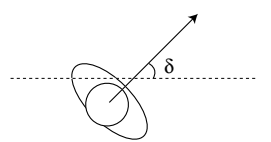

Figure 24. Relation between object and scene coordinate systems

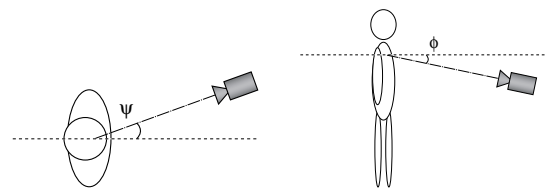

Figure 25. Relation between a virtual camera and an object (II)

\subsection{GUI}

We have also developed a GUI for specifying parameters, which is shown in Figure 26. In this interface, a user can specify the positions, rotations, and scales of the three coordinate systems: the object coordinate system, the background coordinate system, and the virtual camera's. The top, front, and side views of these coordinate systems and the obtained image are displayed in the left top of the window (Figure 26). By specifying the parameters for KFM or ACGM, users can view $2 \mathrm{D}$ image sequences that they desire where the background scene as well as a foreground object are represented in a natural way (Figure 27).

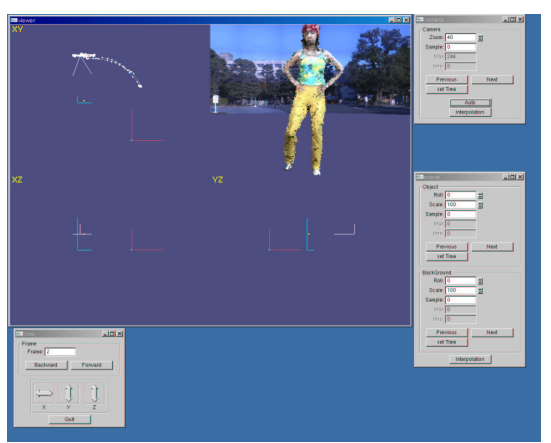

Figure 26. a GUI for 3D video editing and visualization. 


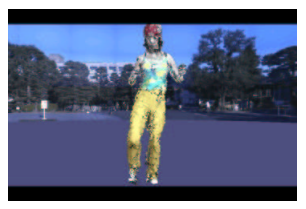

frame \#135

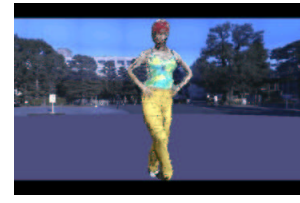

frame \#180

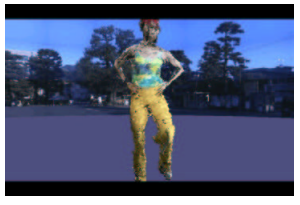

frame \#195

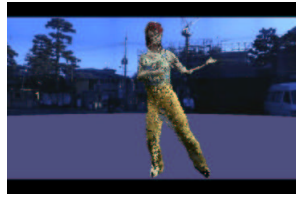

frame \#210

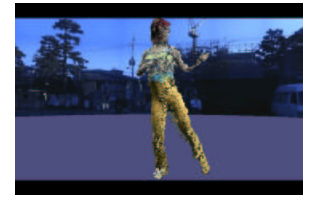

frame \#225

Figure 27. Visualized 3D video with an omnidirectional background

\section{Conclusion}

We are proposing 3D video as new image media: it records the object's full 3D shape, motion, and surface properties (i.e. color and texture). In this paper, following an overview of the real-time 3D object behavior reconstruction system, we proposed a high fidelity texture mapping method and a versatile editing system for 3D video.

The qualitative and quantitative performance evaluations demonstrated that the proposed texture mapping method can produce object images from arbitrary viewpoints in almost the same quality as real video data. With the editing system, moreover, we can generate attractive image contents by employing versatile object and background arrangements in the 3D scene and completely free 3D camera-works.

Based on these novel technologies, we will be able to open up new image media world and promote personal and social activities in education, culture, entertainment, sport, and so on.

To make 3D video usable in everyday life, we still have to develop methods of

- effective data compression

- more natural image generation

- higher speed and more accurate 3D behavior reconstruction

- editing 3D video for artistic image contents.

This work was supported by the grant-in-aid for scientific research (A) 13308017. We are grateful to Real World Computing Partnership, Japan for allowing us to use their multi-viewpoint video data. We also thank Mr. Xiaojun Wu and members of Matsuyama laboratory for their helps and insightful suggestions.

\section{References}

[1] T.Wada, X.Wu, S.Tokai, T.Matsuyama: Homography Based Parallel Volume Intersection: To- ward Real-Time Reconstruction Using Active Camera: CAMP2000 Computer Architectures for Machine Perception, pp.331-339.

[2] E. Borovikov and L. Davis: A Distributed System for Real-Time Volume Reconstruction, Proc. of Computer Architectures for Machine Perception, pp.183-189, 2000.

[3] G.Cheung and T.Kanade: A Real Time System for Robust 3D Voxel Reconstruction of Human Motions, Proc. of CVPR, pp.714-720, 2000.

[4] T.Kanade, P.Rander, S.Vedula, and H.Saito: Virtualized Reality: Digitizing a 3D Time-Varying Event as is and in Real Time, in Mixed Reality (Y.Ohta and H.Tamura eds.), pp.41-57, Ohmsha, 1999.

[5] S.Moezzi, L.Tai, and P.Gerard: Virtual View Generation for 3D Digital Video, IEEE Multimedia, pp.18-26, 1997.

[6] H.Tezuka, A.Hori, Y.Ishikawa, and M.Sato: PM: An Operating System Coordinated High Performance Communication Library, HighPerformance Computing and Networking (P. Sloot and B. Hertzberger, eds.), Lecture Notes in Computer Science, Vol.1225, pp.708-717. Springer-Verlag, 1997.

[7] Matsuyama, T.: "Cooperative Distributed Vision - Dynamic Integration of Visual Perception, Action, and Communication -," Proc. of Image Understanding Workshop, pp. 365-384, 1998

[8] Y.Kenmochi, K.Kotani, A.Imiya: Marching Cubes Method with Connectivity Consideration: PRMU-98-218, pp.197-204, 1999. 